\title{
CONCENTRAÇÕES E ASSOCIAÇÕES GEOQUÍMICAS DE Pb E Zn EM SEDIMENTOS DO RIO SÃo FRANCISCO IMPACTADOS POR REJEITOS DA PRODUÇÃO INDUSTRIAL DE ZINCO
}

\author{
Marcos Vinícius Teles Gomes \\ Estação de Hidrobiologia e Piscicultura de Três Marias, Companhia de Desenvolvimento dos Vales do São Francisco e do Parnaíba, \\ 39205-000 Três Marias - MG, Brasil \\ Adnivia Santos Costa, Carlos Alexandre Borges Garcia, Elisângela Andrade Passos e José do Patrocínio Hora Alves* \\ Departamento de Química, Universidade Federal de Sergipe, Av. Mal. Rondon, s/n, 49100-000 São Cristóvão - SE, Brasil
}

Recebido em 21/6/10; aceito em 28/9/10; publicado na web em 27/10/10

\begin{abstract}
CONCENTRATIONS AND GEOCHEMICAL ASSOCIATIONS OF Pb AND Zn IN SEDIMENTS OF THE RIVER SÃO FRANCISCO IMPACTED BY WASTES FROM INDUSTRIAL ZINC PRODUCTION. Surface sediments from the River São Francisco were analyzed to investigate the impact, due to the presence of metals $(\mathrm{Cd}, \mathrm{Cu}, \mathrm{Pb}, \mathrm{Zn})$ in wastes from a metallurgical industry in the city of Três Marias/MG, Brazil. The concentrations and geochemical associations of $\mathrm{Pb}, \mathrm{Zn}$ and trace metals associated with the minerals employed in zinc production were measured. Sediments close to discharge locations were highly contaminated with $\mathrm{Pb}\left(332-512 \mu \mathrm{g} \mathrm{g}{ }^{-1}\right)$ and $\mathrm{Zn}\left(7872-10780 \mu \mathrm{g} \mathrm{g}^{-1}\right)$, with values decreasing rapidly due to dilution and hydraulic sorting. Evaluation of toxicity according to the Consensus-based Sediment Quality Guidelines indicated for $\mathrm{Cd}, \mathrm{Pb}$ and $\mathrm{Zn}$ a high probability of adverse effects on aquatic biota at these sites.
\end{abstract}

Keywords: contaminated sediment; lead; zinc.

\section{INTRODUÇÃO}

Atividades de mineração e exploração comercial de recursos minerais fazem parte do conjunto das atividades humanas que mais têm contribuído para introdução de metais traço na hidrosfera. Os rejeitos dessas atividades contêm concentrações elevadas de metais e, por causa da instabilidade química e geotécnica desses materiais, representam, por longo tempo, uma fonte potencial de contaminação. ${ }^{1-3}$ A contaminação de sedimentos com metais derivados de efluentes da mineração e do beneficiamento de minérios têm se tornado um problema global $^{4}$ e, por isso, tem adquirido muita importância a avaliação geoquímica desses sedimentos, no sentido de diferenciar o metal derivado daquelas fontes de contaminação daquele originário do background regional e de fontes difusas globais. ${ }^{5,6}$ Tem também sido destacada a necessidade de se entender os processos de dispersão dos metais pelo transporte fluvial, pois isso pode contribuir para melhorar as práticas de gerenciamento dos rios contaminados. ${ }^{7}$

Os sedimentos agem como um reservatório no qual as espécies químicas são acumuladas no decorrer do tempo e, desse modo, se constituem num arquivo útil para avaliação de contaminações passadas. Uma parte significativa dos metais introduzidos no rio por algum aporte, transiente ou contínuo, deve se acumular nos sedimentos através de processos químicos e físicos de sorção. ${ }^{1}$ Vários estudos têm documentado concentrações extremamente elevadas de $\mathrm{Pb}, \mathrm{Zn}$ e $\mathrm{Cd}$ em sedimentos próximos a áreas de mineração, inclusive com a ocorrência de efeitos ecológicos adversos, provocados pelos metais associados aos sedimentos. Nessas mesmas áreas tem também sido registrado aumento das concentrações de metais $(\mathrm{Pb}, \mathrm{Zn}, \mathrm{Cd})$ em invertebrados aquáticos e peixes..$^{8-11}$

As concentrações naturais dos elementos traço em sedimentos são fortemente determinadas pela natureza do material inorgânico resultante do intemperismo físico e químico. Esse material é formado principalmente por um número limitado de silicatos, tais como quartzo, feldspato, micas e argilas minerais, e em menor quantidade por óxi-

\footnotetext{
*e-mail: jalves@ufs.br

\#Artigo em homenagem ao Prof. Hans Viertler
}

dos metálicos e sulfetos. Entre os diferentes substratos geoquímicos que compõem os sedimentos, os mais importantes são aqueles que detêm a capacidade de reter e concentrar elementos traço e nesse caso destacam-se os compostos argilominerais, os óxidos hidróxidos de ferro e manganês e as substancias húmicas e coloides. ${ }^{12-14}$ Portanto, os metais podem estar em diferentes formas ou modos de ligação nos sedimentos: podem precipitar como hidróxidos, sulfetos, carbonatos; serem adsorvidos e/ou coprecipitar com os hidróxidos e óxidos de ferro, manganês e alumínio e formar complexos orgânicos e inorgânicos. ${ }^{15}$

$\mathrm{O}$ zinco é encontrado na natureza principalmente sob a forma de sulfetos mineralizados em rochas calcárias. Na zona de oxidação o minério sulfetado pode sofrer grandes transformações, levando à formação de óxidos, carbonatos e silicatos. No Brasil, os principais minérios de zinco são a esfalerita $(\mathrm{ZnS})$, willemita $\left(\mathrm{Zn}_{2} \mathrm{SiO}_{4}\right)$ e calamina $\left(\mathrm{ZnO} \mathrm{SiO}_{2}\right)$. Cerca de $82,9 \%$ das reservas brasileiras estão localizadas nos municípios de Vazante e Paracatu (Mina de Morro Agudo), região noroeste do estado de Minas Gerais. Em Vazante, o minério é do tipo oxidado (willemita e calamina), com uma capacidade instalada de 70.000 t/ano e em Paracatu a mina tem uma capacidade instalada de 26.000 t/ano de minério sulfetado (esfalerita). ${ }^{16,17}$

Neste trabalho amostras de sedimento superficial foram analisadas para determinação de chumbo e zinco e dos principais metais associados aos minérios usados na produção do zinco, com o objetivo de identificar o impacto provocado no Rio São Francisco pelos rejeitos da usina metalúrgica, localizada na cidade de Três Marias, Minas Gerais, Brasil. Foram investigados também os fatores que controlam a distribuição e mobilidade dos metais nos sedimentos e avaliada a possibilidade da ocorrência de toxicidade, com base nos Valores-Guia Consensual de Qualidade dos Sedimentos, desenvolvido para sedimentos de água doce. ${ }^{18}$

\section{PARTE EXPERIMENTAL}

\section{Área de estudo}

O rio São Francisco nasce no estado de Minas Gerais, região sudeste do Brasil, percorre uma extensão de aproximadamente 2.900 
$\mathrm{km}$, atravessando varias regiões com diversidade em termos de clima, características físicas, ambientais e sociais, até desaguar no Oceano Atlântico na divisa dos estados de Sergipe e Alagoas, região nordeste do país. O rio tem uma vazão média anual de $2.850 \mathrm{~m}^{3} \mathrm{~s}^{-1}$ e a sua bacia hidrográfica cobre uma área de $636.920 \mathrm{~km}^{2} .^{19}$

A área em estudo tem uma extensão aproximada de 42 km e está situada no rio São Francisco entre a barragem do reservatório de Três Marias e a confluência com o rio Abaeté (Figura 1S, material suplementar), na cidade de Três Marias - Minas Gerais. Nessa região está instalada há mais de 50 anos, uma unidade industrial de produção de zinco, que utiliza os depósitos das minas de Morro Agudo e Vazante. ${ }^{20}$ Em Morro Agudo a mineralização do sulfeto com 6,4\% de Zn está associada com calcita, microquartzo, megaquartzo e barita. A mina de Morro Agudo produz também chumbo e como subproduto da flotação, o calcário que é utilizado como corretivo de solos. O minério existente nos depósitos de Vazante é do tipo oxidado, constituído principalmente de willemita com um teor médio de $23 \%$ de $\mathrm{Zn} .^{21,22}$ A planta metalúrgica de Três Marias processa de forma integrada os concentrados silicatados e sulfetados de zinco.

Os rejeitos produzidos pela metalúrgica foram inicialmente lançados diretamente no solo, nas proximidades da área industrial e terminavam sendo carreados para o rio, contaminando a região no entorno do sítio 2. Desde 1983, os resíduos passaram a ser lançados

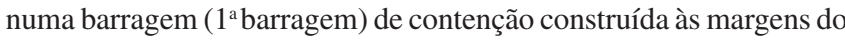
rio, também nas proximidades da indústria e que operou até o ano de 2002. A partir de então, os rejeitos passaram a ser lançados numa nova barragem ( $2^{\mathrm{a}}$ barragem) construída na cabeceira do córrego Lavagem. O córrego Lavagem deságua no córrego Tolda, que é um afluente do rio São Francisco e cuja desembocadura se dá na região a montante do sítio $4 .{ }^{20,23}$ Desse modo, os rejeitos lançados na nova barragem terminam também atingindo, de forma indireta, o rio São Francisco.

\section{Amostragem}

No presente estudo foram coletadas, em dezembro de 2006 (período chuvoso, vazão média de $2.393 \mathrm{~m}^{3} \mathrm{~s}^{-1}$ ) e julho de 2007 (período seco, vazão média de $331 \mathrm{~m}^{3} \mathrm{~s}^{-1}$ ), amostras de sedimentos superficiais, em cinco sítios de amostragem distribuídos como indicado na Figura 1S (material suplementar) e assim discriminados: P1 (18 12' 59,7' S;

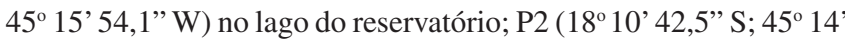

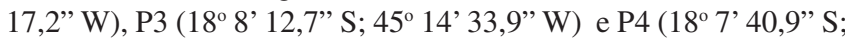
$\left.45^{\circ} 11^{\prime} 25,8^{\prime \prime} \mathrm{W}\right)$ no rio São Francisco próximo à margem direita e, P5 (18 2' 11,9” S; 45 11' 24,7”'W) próximo à margem esquerda do rio São Francisco, nas proximidades da confluência com o rio Abaeté.

Em cada sítio, as amostras de sedimento em triplicata foram tomadas com um busca fundo Van Veen de aço inox. De cada amostra, $5 \mathrm{~cm}$ do topo do sedimento foram removidos, utilizando-se utensílios não metálicos para evitar contaminação, estocados em recipientes plásticos fechados e mantidos em gelo até chegar ao laboratório. Durante o processo de amostragem foi sempre descartada a parte do sedimento que esteve em contato com a parede do amostrador. No laboratório as amostras foram imediatamente secas em estufa de circulação forçada de ar a $50{ }^{\circ} \mathrm{C}$ por $72 \mathrm{~h}$ (até massa constante) e depois homogeneizadas através da moagem em gral de porcelana até se obter um material fino. Em seguida, foram estocadas em frascos de vidro previamente limpos, até serem analisadas.

\section{Análise química}

Para determinação das concentrações dos metais foi utilizado o Método US EPA 200.8. As amostras ( $1 \mathrm{~g})$ foram tratadas em recipientes de Teflon ${ }^{\circledR}$ fechados, com 4 mL de solução de $\mathrm{HNO}_{3}(1: 1)$ e $10 \mathrm{~mL}$ de solução de $\mathrm{HCl}$ (1:5). A mistura foi mantida em sistema fechado por 30 min a $95^{\circ} \mathrm{C}$. Após a extração, as amostras foram filtradas através de filtro de papel quantitativo faixa preta, transferidas para balão volumétrico de $50 \mathrm{~mL}$ e o volume completado com água ultrapura. As soluções foram estocadas em frascos de polietileno para posterior determinação dos metais por espectrometria de absorção atômica em chama ou forno de grafite. Para controle de qualidade, foram preparados e analisados junto com as amostras brancos dos reagentes e o material de referência, sedimento de Lago (LKSD-1 CCNRP/Canadá). Os resultados mostraram não ter ocorrido nenhuma contaminação dos brancos e uma recuperação dos metais $(\mathrm{Cd}, . \mathrm{Cu}$, $\mathrm{Pb}, \mathrm{Zn}, \mathrm{Fe}$ ) no intervalo de $97-103 \%$. Al e Ca não foram medidos no material de referência. As concentrações dos metais foram determinadas em três replicatas.

O carbono total das amostras foi medido num analisador elemen$\operatorname{tar}$ (NCHS-O, Flash ES 1112) com combustão a $900{ }^{\circ} \mathrm{C}$. O carbono orgânico $\left(\mathrm{C}_{\mathrm{org}}\right)$ foi calculado pela diferença do carbono medido antes e após a calcinação das amostras a $550^{\circ} \mathrm{C}$ por $1 \mathrm{~h}$. Para controle de qualidade das análises, foi analisado juntamente com as amostras, o padrão certificado de Sedimento de Lago (LKSD-1 CCNRP/ Canadá) obtendo-se, em três replicatas, uma recuperação média de $96,3 \pm 4,2 \%$.

Os cálculos dos coeficientes de correlação foram feitos usando-se o programa Statistica for Windows Versão 6.0.

\section{RESULTADOS E DISCUSSÃO}

\section{Variações nas concentrações dos metais nos sedimentos}

As concentrações dos metais traço $(\mathrm{Cd}, \mathrm{Cu}, \mathrm{Pb}, \mathrm{Zn})$ carbono orgânico, ferro, alumínio e cálcio, nas amostras dos sedimentos coletados a montante dos rejeitos (P1, pré-rejeitos), na área de influência dos rejeitos (P2, P3, P4) e após a confluência com o rio Abaeté (P5, pós-rejeitos) são mostradas na Tabela 1.

Assumindo que os sedimentos do reservatório não são afetados pelas atividades e rejeitos da mineração, as concentrações médias ( $\mu \mathrm{g}$ $\left.\mathrm{g}^{-1}\right)$ de $\mathrm{Zn}(8,48), \mathrm{Pb}(10,50), \mathrm{Cu}(1,36)$ e Cd $(0,02)$ medidas em P1 foram consideradas o background da região. Os sedimentos do sítio P2 apresentaram as concentrações mais elevadas para os metais traço. Esse sítio encontra-se próximo ao local de descarga dos efluentes da unidade industrial, estando diretamente impactado pelos rejeitos, que inicialmente eram lançados no solo e depois numa barragem de contenção e que terminavam atingindo o rio, tanto pela ação das chuvas sobre o solo, como pelo transbordamento e/ou vazamento da barragem. Nas proximidades desse mesmo sítio, Oliveira, ${ }^{20} \mathrm{em} 2003$, também encontrou valores elevados para Cd (7-23 $\left.\mu \mathrm{g} \mathrm{g}^{-1}\right), \mathrm{Cu}(24-122$ $\left.\mu \mathrm{g} \mathrm{g}^{-1}\right), \mathrm{Pb}\left(44-380 \mu \mathrm{g} \mathrm{g}^{-1}\right)$ e Zn $\left(655->5000 \mu \mathrm{g} \mathrm{g}^{-1}\right)$. Em P3, embora as concentrações sejam elevadas em relação a P1, foram muito inferiores quando comparadas com os valores de P2. As concentrações dos metais nos sedimentos do sítio $\mathrm{P} 4$, localizado próximo à confluência com o córrego Tolda, voltaram a crescer significativamente, embora ainda sejam menores que em P2. O córrego Tolda, também conhecida como ribeirão Espírito Santo, sofre influência indireta de uma outra barragem de rejeito da mesma unidade industrial, implantada em 2002 nas margens do córrego Lavagem. ${ }^{20}$ Em P5 as concentrações retornam ao nível de background da região, com valores equivalentes aos medidos em P1.

Observa-se, portanto, que as concentrações dos metais traço foram mais elevadas nas amostras dos sedimentos coletadas nos sítios próximos às fontes de contaminação $(\mathrm{P} 2, \mathrm{P} 4)$. No período úmido, em geral, as concentrações foram mais elevadas como consequência da maior quantidade de material contaminado, carreado pelo elevado deflúvio superficial.

Em sistemas fluviais as concentrações dos metais tendem a de- 
Tabela 1. Concentrações dos metais, carbono orgânico ( $\mathrm{n}=3$, média \pm desvio padrão), relações Pb:Zn em sedimentos superficiais do Rio São Francisco/Três Marias-MG no período seco (S) e chuvoso (C), e valores-guia consensual para sedimentos de água doce (TEC, concentração de efeito limiar; PEC, concentração de efeito provável)

\begin{tabular}{|c|c|c|c|c|c|c|c|c|c|}
\hline Sítio & $\mathrm{Cd} /\left(\mu \mathrm{g} \mathrm{g}^{-1}\right)$ & $\mathrm{Cu} /\left(\mu \mathrm{g} \mathrm{g}^{-1}\right)$ & $\mathrm{Pb} /\left(\mu \mathrm{g} \mathrm{g}^{-1}\right)$ & $\mathrm{Zn} /\left(\mu \mathrm{g} \mathrm{g}^{-1}\right)$ & $\mathrm{Ca} /\left(\mu \mathrm{g} \mathrm{g}^{-1}\right)$ & $\mathrm{Fe} /(\%)$ & $\mathrm{Al} /(\%)$ & Corg/(\%) & $\mathrm{Pb} / \mathrm{Zn}$ \\
\hline $\mathrm{P} 1 \mathrm{C}$ & $0,02 \pm 0,01$ & $1,3 \pm 0,1$ & $10,5 \pm 0,5$ & $8,6 \pm 1,2$ & $5,5 \pm 0,4$ & $0,3 \pm 0,1$ & $0,7 \pm 0,2$ & $0,10 \pm 0,02$ & 1,20 \\
\hline $\mathrm{P} 2 \mathrm{C}$ & $19,90 \pm 0,38$ & $93,9 \pm 7,2$ & $512 \pm 35$ & $10780 \pm 940$ & $487 \pm 36$ & $0,8 \pm 0,1$ & $2,3 \pm 0,3$ & $0,21 \pm 0,03$ & 0,05 \\
\hline $\mathrm{P} 3 \mathrm{C}$ & $0,08 \pm 0,01$ & $6,3 \pm 0,2$ & $28,2 \pm 2,1$ & $78,6 \pm 5,1$ & $38,0 \pm 4,6$ & $2,5 \pm 0,2$ & $9,1 \pm 0,8$ & $0,97 \pm 0,06$ & 0,40 \\
\hline $\mathrm{P} 4 \mathrm{C}$ & $10,68 \pm 0,21$ & $16,1 \pm 1,0$ & $62,5 \pm 5,2$ & $1706 \pm 121$ & $25,4 \pm 1,8$ & $1,5 \pm 0,1$ & $4,9 \pm 0,7$ & $1,50 \pm 0,03$ & 0,04 \\
\hline P5C & $0,02 \pm 0,01$ & $1,3 \pm 0,1$ & $10,8 \pm 0,7$ & $8,1 \pm 0,6$ & $22,5 \pm 2,1$ & $0,5 \pm 0,1$ & $1,5 \pm 0,1$ & $0,16 \pm 0,02$ & 1,32 \\
\hline $\mathrm{P} 1 \mathrm{~S}$ & $0,03 \pm 0,02$ & $1,4 \pm 0,2$ & $10,5 \pm 0,4$ & $8,4 \pm 0,7$ & $4,4 \pm 0,7$ & $1,0 \pm 0,2$ & $3,7 \pm 0,5$ & $0,04 \pm 0,01$ & 1,25 \\
\hline $\mathrm{P} 2 \mathrm{~S}$ & $6,91 \pm 0,19$ & $34,3 \pm 3,6$ & $332 \pm 24$ & $7872 \pm 652$ & $221 \pm 45$ & $0,5 \pm 0,1$ & $1,6 \pm 0,2$ & $0,09 \pm 0,02$ & 0,04 \\
\hline P3S & $3,94 \pm 0,26$ & $8,2 \pm 0,5$ & $33,2 \pm 2,6$ & $1076 \pm 85$ & $21,6 \pm 3,0$ & $0,6 \pm 0,1$ & $1,6 \pm 0,2$ & $0,47 \pm 0,01$ & 0,31 \\
\hline $\mathrm{P} 4 \mathrm{~S}$ & $3,60 \pm 0,18$ & $12,6 \pm 1,2$ & $78,7 \pm 4,7$ & $1076 \pm 124$ & $25,0 \pm 2,0$ & $0,7 \pm 0,2$ & $2,3 \pm 0,2$ & $1,13 \pm 0,02$ & 0,07 \\
\hline P5S & $0,03 \pm 0,01$ & $1,4 \pm 0,1$ & $9,5 \pm 0,4$ & $8,9 \pm 0,8$ & $17,4 \pm 3,1$ & $0,5 \pm 0,1$ & $1,5 \pm 0,1$ & $0,06 \pm 0,01$ & 1,07 \\
\hline TEC & 0,99 & 31,6 & 35,8 & 121 & & & & & \\
\hline PEC & 4,98 & 149 & 128 & 459 & & & & & \\
\hline
\end{tabular}

crescer nos sedimentos dos sítios que ficam a jusante das fontes de contaminação, é isso o que se observa em P3. Esse padrão tem sido atribuído a processos hidrodinâmicos, como a diluição com sedimentos não contaminados transportados pelo rio e a seleção hidráulica durante o transporte dos sedimentos. ${ }^{24-26} \mathrm{~A}$ importância dos processos fluviais na dispersão dos metais a partir de sítios contaminados é bem conhecida, o sedimento em suspensão transportado pelo rio é um processo contínuo e de longe, o mais importante em relação à dispersão, principalmente nos períodos de elevada vazão. ${ }^{26,27}$ Considerando ainda que o sedimento em suspensão é composto tipicamente de silte e argila, a dispersão através do material em suspensão se dá de forma muito mais rápida e mais extensa do que através do material de fundo, formado principalmente por partículas de areia ou maiores. ${ }^{28}$

As relações entre as concentrações de $\mathrm{Pb}: \mathrm{Zn}$ (Tabela 1) em P1 (1,20-1,25) e em P5 (1,07-1,32) foram equivalentes, confirmando assim que o material contaminado não deve atingir de forma significativa o sítio P5. Nos sedimentos dos sítios diretamente afetados pelos rejeitos da unidade industrial, as relações foram 0,04-0,05 em P2 e 0,04-0,07 em P4, o que reflete a origem comum da fonte de contaminação dos dois sítios. Em P3, como consequência do processo de seleção hidráulica, a razão $\mathrm{Pb}: \mathrm{Zn}$ foi aumentada para 0,31-0,40, indicando um relativo enriquecimento do chumbo sobre o zinco. Hillier et al ${ }^{28}$ fazendo análise para determinação das formas de $\mathrm{Pb}$ em diferentes frações do sedimento em suspensão, numa área de mineração, encontraram concentrações maiores nas frações mais finas e uma grande proporção do $\mathrm{Pb}$ presente na forma de cerussite $\left(\mathrm{PbCO}_{3}\right)$. Essa distribuição evidencia a grande probabilidade do $\mathrm{Pb}$ ser mobilizado em maior extensão, principalmente pelos processos que afetam o transporte e a deposição do material fino em suspensão.

\section{Associações dos metais nos sedimentos}

A análise de correlação foi utilizada para identificar os fatores potenciais que controlam a distribuição e mobilidade dos metais nos sedimentos. A matriz de correlação de Spearman é mostrada na Tabela 2. Os metais $\mathrm{Zn}, \mathrm{Pb}, \mathrm{Cu}, \mathrm{Cd}$ e $\mathrm{Ca}$ foram escolhidos pelas suas presenças nos minérios processados. $\mathrm{O} \mathrm{Pb}$ está presente de forma substancial na galena e o zinco na esfalerita e willemita. Em menor proporção também aparecem associados aos minérios $\mathrm{Cu}$ e Cd. No minério de $\mathrm{Zn}$ e $\mathrm{Pb}$ da mina de Morro Agudo, a rocha hospedeira é um calcário dolomítico, por isso o Ca foi também selecionado, no sentido de se obter informações sobre a participação do $\mathrm{CaCO}_{3}$ na distribuição dos metais traço nos sedimentos estudados. Foram também incluídos Al, Fe e Corg, como indicadores dos substratos geoquímicos mais importantes na retenção e concentração de elementos traço, tais como, os compostos argilominerais, os óxidos hidróxidos de ferro e manganês e as substâncias húmicas e coloides.

Tabela 2. Matriz de correlação de Spearman para as amostras de sedimentos superficiais do Rio São Francisco/Três Marias-MG (n=5, p<0,05)

\begin{tabular}{lllllllll}
\hline & $\mathrm{Cd}$ & $\mathrm{Cu}$ & $\mathrm{Pb}$ & $\mathrm{Zn}$ & $\mathrm{Ca}$ & $\mathrm{Fe}$ & $\mathrm{Al}$ & $\mathrm{C}_{\text {org }}$ \\
\hline $\mathrm{Cd}$ & 1,00 & & & & & & & \\
$\mathrm{Cu}$ & 0,93 & 1,00 & & & & & & \\
$\mathrm{~Pb}$ & 0,86 & 0,96 & 1,00 & & & & & \\
$\mathrm{Zn}$ & 0,85 & 0,94 & 0,99 & 1,00 & & & & \\
$\mathrm{Ca}$ & 0,86 & 0,98 & 0,98 & 0,96 & 1,00 & & & \\
$\mathrm{Fe}$ & $-0,00$ & $-0,06$ & $-0,14$ & $-0,14$ & $-0,10$ & 1,00 & & \\
$\mathrm{Al}$ & $-0,05$ & $-0,09$ & $-0,16$ & $-0,16$ & $-0,12$ & 1,00 & 1,00 & \\
$\mathrm{C}_{\text {org }}$ & 0,17 & $-0,09$ & $-0,16$ & $-0,19$ & $-0,23$ & 0,62 & 0,58 & 1,00 \\
\hline
\end{tabular}

Os metais traço não apresentaram uma correlação com Corg, indicando uma fraca associação ou não associação desses elementos com a matéria orgânica do sedimento. Isso confirma a observação de que em sedimentos não marinhos, o Corg não é um carreador tão forte como são as argilas e os óxidos metálicos. ${ }^{14,29}$ Também não foi observada uma correlação com o alumínio e o ferro, evidenciando que os carreadores inorgânicos tradicionais como as argilas e os óxidos metálicos não determinam as concentrações dos metais traço nos sedimentos contaminados.

As concentrações de $\mathrm{Zn}, \mathrm{Pb}, \mathrm{Cu}$ e $\mathrm{Cd}$ foram fortemente correlacionadas entre si e com o Ca, indicando que esses metais são originários de uma mesma fonte. Considerando que o carbonato de cálcio está presente numa proporção expressiva nos minérios processados, fica evidente que os efluentes da usina de produção de zinco devem ser a principal fonte desses metais para os sedimentos contaminados. Nesse caso, os metais foram lixiviados, adsorvidos ao $\mathrm{Ca}(\mathrm{OH})_{2}$ e $\mathrm{CaCO}_{3}$ em suspensão no efluente, coprecipitando posteriormente no sedimento do rio.

Apesar dos carbonatos não serem considerados um componente eficiente na acumulação de metais traço, vários trabalhos têm mos- 
trado que a correlação com o cálcio, obtida para sedimentos impactados por rejeitos de mineração e sedimentos ricos em carbonato, é provavelmente resultado da adsorção e coprecipitação dos metais sobre o $\mathrm{Ca}(\mathrm{OH})_{2}$ e $\mathrm{CaCO}_{3}$ em suspensão. ${ }^{30-33}$

\section{Toxicidade dos sedimentos}

Os metais nos sedimentos estão distribuídos em fases, não totalmente caracterizadas, tais como carbonatos, óxidos de ferro e manganês, matéria orgânica, sulfetos e argilas, e podem ser redispostas para a água em decorrência de mudanças ambientais. Assim, a toxicidade do metal é influenciada pela forma de ligação no sedimento e não somente pela taxa de acumulação, pois apenas uma fração do conteúdo total é biodisponivel. ${ }^{34}$

Os Valores-Guia de Qualidade dos Sedimentos (VGQS) têm sido usados como ferramentas de referência para avaliar o grau em que os metais associados aos sedimentos podem afetar adversamente os organismos aquáticos. ${ }^{35} \mathrm{O}$ desenvolvimento e a implementação desses valores refletem o reconhecimento da importância dos sedimentos contaminados como uma possível causa de efeitos ambientais adversos. ${ }^{36}$ Embora tenham sido desenvolvidos para a América do Norte, os índices estabelecidos pelos VGQSs vêm sendo usados para avaliação da concentração de contaminantes nos sedimentos em várias regiões do mundo. . $^{14,34,37,38}$

$\mathrm{Na}$ ausência de informações ecotoxicológicas, foi usado, neste estudo, o VGQS ${ }^{18}$ consensual (Consensus-based Sediment Quality Guidelines) desenvolvido para sedimentos de água doce, a fim de avaliar as concentrações de $\mathrm{Cd}, \mathrm{Cu}, \mathrm{Pb}$ e $\mathrm{Zn}$, quanto à possibilidade de ocorrência de efeitos adversos para os organismos aquáticos. A Tabela 1 mostra, além das concentrações obtidas para cada metal, a concentração de efeito limiar (TEC, threshold effect concentration), abaixo da qual efeitos adversos não devem ocorrem, e a concentração de efeito provável (PEC, probable effect concentration), acima da qual efeitos adversos podem ocorrem frequentemente. Em P1 e P5 as concentrações ficaram muito abaixo do valor do TEC, não devendo nesses sítios ocorrer efeitos adversos para os organismos aquáticos. Em P2, tanto no período seco como úmido, as concentrações de $\mathrm{Cd}$, $\mathrm{Pb}$ e $\mathrm{Zn}$ excederam o valor do PEC. Em P3, no período úmido as concentrações ficaram abaixo do TEC e no período seco os valores de $\mathrm{Cd}$ e $\mathrm{Zn}$ foram superiores ao TEC e PEC, respectivamente. Em $\mathrm{P} 4$, no período úmido as concentrações de $\mathrm{Cd}$ e $\mathrm{Zn}$ foram superiores ao PEC e o $\mathrm{Pb}$ superior ao TEC. No período seco os valores de $\mathrm{Cd}$ e $\mathrm{Pb}$ ficaram acima do TEC e o $\mathrm{Zn}$ acima do PEC. As concentrações de $\mathrm{Cu}$, em geral, foram inferiores ao TEC, excedendo esse limite apenas no sítio P2. Portanto, fica evidente que $\mathrm{Cd}, \mathrm{Pb}$ e $\mathrm{Zn}$, nos sedimentos dos sítios $\mathrm{P} 2$ e P4, devem apresentar com frequência efeitos adversos para a biota aquática. A probabilidade de incidência desses efeitos é muito elevada, considerando-se que a capacidade de predição do PEC foi de $94 \%$ para o Cd e de $90 \%$ para $\mathrm{Pb}$ e $\mathrm{Zn} .{ }^{18}$ Vale ressaltar, no entanto, que os VGQS devem ser usados com cautela, pois não foram desenvolvidos especificamente para a biogeoquímica da região em estudo. Por isso, para uma maior segurança é importante a validação dos resultados aqui obtidos através de testes de toxicidade.

\section{CONCLUSÕES}

Neste trabalho foram investigadas a distribuição, as associações geoquimicas e o risco de toxicidade para $\mathrm{Cd}, \mathrm{Cu}, \mathrm{Pb}$ e $\mathrm{Zn}$ em sedimentos superficiais do rio São Francisco, na região impactada pelos rejeitos da indústria metalúrgica de zinco na cidade de Três Marias/ MG, Brasil. Os sedimentos próximos aos locais de descarte foram altamente contaminados com Cd (6,9-10,9 $\left.\mu \mathrm{g} \mathrm{g}^{-1}\right), \mathrm{Cu}(34,3-93,9 \mu \mathrm{g}$ $\left.\mathrm{g}^{-1}\right), \mathrm{Pb}\left(332-512 \mu \mathrm{g} \mathrm{g}^{-1}\right)$ e $\mathrm{Zn}\left(7872-10780 \mu \mathrm{g} \mathrm{g}^{-1}\right)$, declinando esses valores rapidamente e retornando em P5 aos níveis medidos em P1, considerados como background da região. O padrão de distribuição observado, com decréscimo das concentrações nos sítios que ficam a jusante das fontes de contaminação é coerente com o que tem sido descrito para sedimentos de áreas próximas a locais de mineração.

A matriz de correlação mostrou que os carreadores tradicionais (Al, Fe, Corg) não determinam as concentrações dos metais traço nos sedimentos analisados. $\mathrm{Zn}, \mathrm{Pb}, \mathrm{Cu}$ e $\mathrm{Cd}$ foram fortemente correlacionados entre si e com o $\mathrm{Ca}$, evidenciando que os rejeitos da usina metalúrgica devem ser a fonte comum desses metais para os sedimentos contaminados. O carbonato de cálcio foi o principal componente na acumulação do $\mathrm{Zn}, \mathrm{Pb}, \mathrm{Cu}$ e $\mathrm{Cd}$, através da adsorção e coprecipitação desses metais sobre o $\mathrm{Ca}(\mathrm{OH})_{2} \mathrm{e} \mathrm{CaCO}_{3}$ em suspensão.

A toxicidade avaliada com base nos Valores-Guia Consensual de Qualidade dos Sedimentos mostrou que, nos sedimentos dos sítios P2 e P4, existe uma probabilidade elevada de incidência de efeitos adversos para a biota aquática, associada às concentrações de $\mathrm{Cd}, \mathrm{Pb}$ e $\mathrm{Zn}$.

\section{MATERIAL SUPLEMENTAR}

Disponível em http://quimicanova.sbq.org.br, na forma de arquivo .PDF, com acesso livre.

\section{REFERÊNCIAS}

1. Ferreira da Silva, E.; Almeida, S. S. P.; Nunes, M. L.; Luís, A. T.; Borg, F.; Hedlund, M.; Sá, C. M.; Patinha, C.; Teixeira, P.; Sci. Total Environ. 2009, 407, 5620 .

2. Ferreira da Silva, E.; Patinha, C.; Reis, P.; Cardoso Fonseca, E.; Matos, J. X.; Barrosinho, J.; Santos Oliveira, J. M.; Environ. Geol. 2006, 50, 1001.

3. Sánchez España, J.; Pamo, E. I.; Santofimia, E.; Aduvire, O.; Reyes, J.; Barettino, D.; Appl. Geochem. 2005, 20, 1320.

4. Pulford, I. D.; MacKenzie, A. B.; Donatello, S.; Hastings, L.; Environ. Pollut. 2009, 157, 1649.

5. MacKenzie, A. B.; Pulford, I. D.; Appl. Geochem. 2002, 17, 1093.

6. Macklin, M. G.; Brewer, P. A.; Hudson-Edwards, K. A.; Bird, G.; Coulthard, T. J.; Dennis, I. A.; Lechler, P. J.; Miller, J. R.; Turner, J. N.; Geomorphology 2006, 79, 423.

7. Carter, J.; Walling, D. E.; Owens, P. N.; Leeks, G. J. L.; Hydrol. Process. 2006, 20, 3007.

8. Besser, J. M.; Brumbaugh, W. G.; May, T. W.; Schmitt, C. J.; Environ. Monit. Assess. 2007, 129, 227.

9. Schmitt, C. J.; Brumbaugh, W. G.; May, T. W.; Ecotoxicol. Environ. Saf. 2007, 67, 14.

10. Besser, J. M.; Brumbaugh, W. G.; Allert, A. L.; Poulton, B. C.; Schmitt, C. J.; Ingersoll, C. G.; Ecotoxicol. Environ. Saf. 2009, 72, 516.

11. Larsen, T. S.; Kristensen, J. A.; Asmund, G.; Bjerregaard, P.; Environ. Pollut. 2001, 114, 275.

12. Forstner, U.; Salomons, W.; Environ. Technol. Lett. 1980, 1, 494.

13. Windom, H. L.; Schopp, S. J.; Calder, F. D.; Ryan, J. D.; Smith Jr., R. G.; Burney, L. C.; Lewis, F. G.; Rawlinson, C. H.; Environ. Sci. Technol. 1989, 23, 314 .

14. Garcia, C. A. B.; Barreto, M. S.; Passos, E. A.; Alves, J. P. H.; J. Braz. Chem. Soc. 2009, 20, 1334.

15. Marengo, E.; Gennaro, M. C.; Robotti, E; Rossanigo, P.; Rinaudo, C.; Roz-Gastaldi, M.; Anal. Chem. Acta 2006, 560, 172.

16. DNPM - Departamento Nacional de Produção Mineral; Balanço Mineral Brasileiro 2001, http://www.dnpm.gov.br/assets/galeriadocumento/ balancomineral2001/ zinco.pdf, acessada em Maio 2010.

17. DNPM - Departamento Nacional de Produção Mineral; Sumário Mineral 2005, http://www.dnpm.gov.br/assets/galeriadocumento/ sumariomineral2005/ZINCO\%202005rev.doc, acessada em Maio 2010. 
18. MacDonald, D. D.; Ingersoll, C. G.; Berger, T. A.; Arch. Environ. Contam. Toxicol. 2000, 39, 20.

19. OES - Organization of American States; São Francisco Basin, http:// www.oas.org/dsd/events/english/documents/OSDE_4SaoFrancisco.pdf, acessada em Maio 2010.

20. Oliveira, M. R.; Tese de Doutorado, Universidade Federal de Minas Gerais, Brasil, 2007.

21. Misi, A.; Iyer, S. S. S.; Coleho, C. E. S.; Tassinari, C. C. G.; FrancaRocha, W. J. S.; Cunha, I. A.; Gomes, A. S. R.; Oliveira, T. F.; Teixeira, J. B.; Filho, V. M. C.; Ore Geology Reviews 2005, 26, 263.

22. Monteiro, L. V. S.; Bettencourt, J. S.; Spiro, B.; Graça, R.; Oliveira, T. F.; Exploration and Mining Geology 1999, 8, 21.

23. Saraiva, V. K.; Nascimento, M. R. L.; Palmieri, H. E. L.; Jacomino, V. M. F.; Quim. Nova 2009, 32, 1995.

24. Muller, G.; Forstner, U.; Environ. Geol. 1977, 1, 33.

25. Yim, W. W. S.; Environ. Geol. 1981, 3, 245.

26. Hudson-Edwards, K. A.; Macklin, M. G.; Curtis, C. D.; Vaughan, D. J.; Environ. Sci. Technol. 1996, 30, 72.

27. Walling, D. E.; Moorehead, P. W.; Hydrobiologia 1989, 176/177, 125.
28. Hillier, S.; Suzuki, K.; Cotter-Howells, J.; Appl. Geochem. 2001, 16 , 597.

29. Liaghati, T.; Preda, M.; Cox, M.; Environ. Int. 2003, 29, 935.

30. Bilinski, H.; Kozar, S.; Plavsic, M.; Kwokal, Z.; Branica, M.; Mar. Chem. 1991, 32, 225.

31. Kozar, S.; Bilinski, H.; Branica, M.; Mar. Chem. 1992, 40, 215.

32. Surija, B.; Branica, M.; Sci. Total Environ. 1995, 170, 101.

33. von der Heyden, C. J.; New, M. G.; J. Geochemical Exploration 2004, 82,35 .

34. Bacon, J. R.; Davidson, C. M.; Analyst 2008, 133, 25.

35. Caeiro, S.; Costa, M. H.; Ramos, T. B.; Fernandes, F.; Silveira, N.; Coimbra, A.; Medeiros, G.; Painho, M.; Ecol. Indic. 2005, 5, 151

36. Mil-Homens, M.; Stevens, R. L.; Abrantes, F.; Cato, I.; Cont. Shelf Res. 2006, 26, 1184

37. Chatterjee, M.; Silva Filho, E. V.; Sarkar, S. K.; Sella, S. M.; Bhattacharya, A.; Satpathy, K. K.; Prasad, M. V. R.; Chakraborty, S.; Bhattacharya, B. D.; Environ. Int. 2007, 33, 346.

38. Hortellani, M. A.; Sarkis, J. E. S.; Abessa, D. M. S.; Souza, E. C. P. M.; Quim. Nova 2008, 31, 10. 